\title{
ANALYSIS OF EMERGENCY HELPLINE SUPPORT FOR HOME VENTILATOR DEPENDENT PATIENTS: RISK MANAGEMENT AND WORKLOAD
}

\section{Chatwin, S. Heather, A. Hanak, M.I. Polkey, B. Wilson and A.K. Simonds}

Noninvasive Ventilation Award, sponsored by ResMed

Clinical and Academic Dept of Sleep and Breathing, Royal Brompton Hospital, London, UK

WINNING ABSTRACT: From a total of 1211 adult \& paediatric patients receiving home ventilation (HV) supervised by Royal Brompton Hospital between 1/1/06 and 30/6/06 the respiratory support team received an average of 528 daytime calls/month and $14 /$ month out of hours calls to a telephone helpline.

Diagnoses included: neuromuscular disease, chest wall disease, COPD, obesity hypoventilation and non-COPD lung disease. $99 \%$ received non-invasive ventilation, 1\% tracheostomy ventilation. 149 required 2 ventilators for near 24 hour ventilator dependency, the remainder were classified as $1(17 \%) 2(33 \%) \& 3(50 \%)$ night dependency as were able to breathe spontaneously for this period. $50 \%$ used bilevel positive pressure ventilators, $48 \%$ inspiratory pressure ventilators and $2 \%$ volume ventilators. In 188 calls a home visit was carried out because of ventilator or associated equipment-related problems. Despite regular equipment servicing programme, in 188 patients there was a technical problem with the equipment which was solved in the patient's home in $64 \%$ or required replacement / parts in $22 \%$. Of the 25 calls in which no fault was found, 13 patients were unwell at home or required hospital admission, 2 patients died within 1 month of identification of no fault. No patient was admitted as a result of technical failure of equipment.

Conclusion: There is a significant workload associated with supporting HV patients. Patients / carers all received standard competency training before discharge but other calls may be reduced by a more flexible problem-solving approach. Importantly, reports in which no technical fault is found may indicate deteriorating health in the patient and require close follow-up.

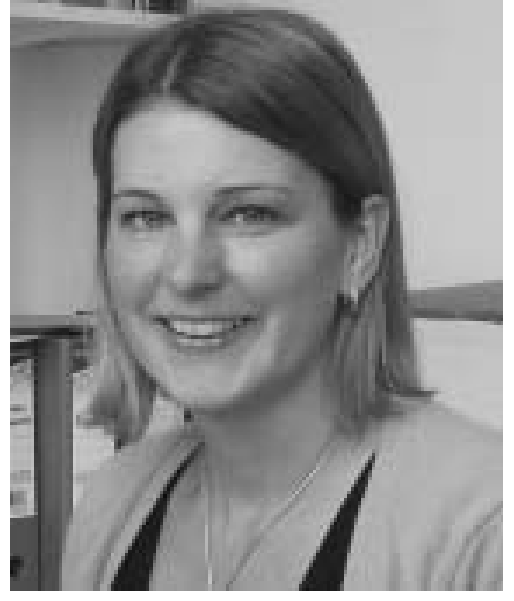

Michelle Chatwin

Clinical and Academic Dept of Sleep and Breathing, Royal Brompton Hospital, London, UK

\section{MY JOB AND THE UNIT IN WHICH I WORK}

I work as a Clinical Specialist Physiotherapist at the Clinical and Academic Dept of Sleep and Breathing at the Royal Brompton Hospital, London, UK. The Clinical and Academic Dept of Sleep and Breathing at the Brompton Hospital has provided a national service for the assessment of sleep and ventilation for $>10 \mathrm{yrs}$ under the leadership of Dr Anita Simonds.

STATEMENT OF INTEREST: M. Chatwin received an ERS travel grant in 2006 and received a reimbursement at the 2007 ERS Congress. Breas Medical (Sweden) contributes funds to pay $95 \%$ of M. Chatwin's salary.
The dept at the Brompton Hospital is able to offer a one-stop service whereby patients can undergo the following: sleep study, assessment of sleepiness/wakefulness tests, lung function, blood gases, respiratory muscle tests, and ventilatory drive; these can all be assessed at one centre and on one visit.

The unit specialises in performing and interpreting all types of sleep study in both adults and paediatric patients. This service is unique in that equipment, expertise and personnel are available to perform assessments across the age range. Although some tests are more suited to adults than children, a full assessment of sleep, ventilation and respiratory muscle function can be performed in all age groups.

The dept has a long history of research in areas allied to noninvasive ventilation (NIV), sleep disorders, respiratory muscle physiology, pulmonary rehabilitation, scoliosis, neuromuscular disease (NMD) and chronic obstructive pulmonary disease. My role includes managing the clinical referrals and carrying out research in NIV and airway clearance in patients with NMD.

\section{MY WINNING POSTER AS PART OF MY RESEARCH}

My PhD is entitled "Respiratory muscle weakness and cough augmentation in patients with hereditary neuromuscular disease". The development of significant respiratory muscle weakness is an integral feature in certain childhood-onset NMD, e.g. spinal muscular atrophy, Duchenne muscular dystrophy and congenital muscular dystrophy. This can lead to the development of nocturnal hypoventilation followed by daytime ventilatory failure and/or respiratory tract infections. Both factors are a major cause of morbidity and mortality in the NMD population [1]. 
Patients with spinal muscular atrophy may be more prone to respiratory tract infection before the development of nocturnal hypoventilation. I have evaluated respiratory muscle strength in a group of spinal muscular atrophy patients and compared it with age-matched patients with Duchenne muscular dystrophy and healthy controls [2]. Spinal muscular atrophy patients had weaker expiratory muscles and often the muscle strength was below the threshold for an effective cough, while inspiratory muscle strength was above the threshold associated with nocturnal hypercapnia.

Mechanical insufflation/exsufflation is a technique that has been claimed to improve cough strength in neuromuscular patients; however, there are no published data prospectively evaluating this device. I have investigated the effect of mechanical insufflation/exsufflation plus other cough augmentation techniques on peak cough flow [3]. The results of this study established that mechanical insufflation/exsufflation had the greatest effect on peak cough flow: I assessed the usefulness of mechanical insufflation/exsufflation when compared with conventional physiotherapy in these NMD patients during an acute respiratory tract infection. It was found that mechanical insufflation/exsufflation decreased the amount of treatment time required to clear secretions compared with conventional treatment [4].

NIV has been shown to improve prognosis and quality of life in NMD patients [5]. I have evaluated the initiation of NIV provision both as an outpatient and as an inpatient. The results of this study suggest that initiation of NIV does not have to be carried out exclusively in the hospital environment, as comparable results are seen with in-patient and outpatient initiation of NIV [6]. A key area after the initiation of NIV is discharge information, risk management and workload. By evaluating the telephone calls to our service and ventilator failure reported to our service, it was found that there is a significant workload associated with the continued care and risk management of home-ventilation patients. Ventilator malfunction was more common in those patients who used their ventilators for $>12 \mathrm{~h} \cdot \mathrm{day}^{-1}$. Patients/carers all received standard competency training before discharge, but other calls may be reduced by a more flexible problem-solving approach. Importantly, reports in which no technical fault is found with the ventilatory equipment may indicate deteriorating health in the patient and require close follow-up.

\section{MY RESEARCH AS PART OF MY WORKING GROUP/ RESEARCH TEAM}

My research fits well into the main research area of our unit. I am part of a wider team from the Clinical and Academic Dept of Sleep and Breathing. The Clinical and Academic Dept of Sleep and Breathing consists of the following three respiratory physicians: Dr Anita Simonds, Professor Michael Polkey and Dr Matthew Hind, and a Reader in respiratory physiology, Dr Mary Morrell. The clinical team consists of five specialist allied health professionals and six research fellows. Key areas of research in our dept are as follows: to investigate the causes and consequences of breathing disorders that occur during sleep; and to translate our research into improvements in patient care. Projects include investigations into how problems of breathing during sleep affect the heart and brain, particularly in older people and patients with pre-existing heart disease, such as heart failure. We are also studying the best way to treat patients with NIV [6]. We have shown that an innovative auto-titrating NIV is as effective as an expertly set up standard ventilator in controlling nocturnal hypoventilation in a randomised crossover study in restrictive chest wall and neuromuscular patients. In a European Respiratory Society Task Force, we have examined end-of-life decisionmaking in severe lung disease patients in high-dependency units in Europe and compared findings with withholding and withdrawing therapy decisions made in intensive care units [7]. We have shown that sleep apnoea is prevalent in patients with both severe and mild heart failure; this finding has raised awareness amongst cardiologists and we are now seeing more referrals. In addition, in heart failure patients with sleep apnoea, we have evaluated the use of heart rate variability. The advantage of this methodology is that 24-h ECG is established in most cardiology departments and patients are familiar with it; it is therefore likely to be cheaper and better tolerated than more complex respiratory investigations. To assess this observation in more detail, we are starting a large study, funded by the British Heart Foundation, to establish the most efficient way to diagnose sleep apnoea in heart failure patients.

\section{THE IMPACT OF MY WORK ON CLINICAL OR RESEARCH PRACTICE}

In summary, my research has provided novel clinical information on where and how noninvasive ventilation can be initiated, along with the impact on workload and risk management. This has allowed our dept to improve and formalise education and discharge planning for patients requiring noninvasive ventilation. We are able to identify patients who may be at risk of an acute exacerbation if ventilator failure is reported and no fault is found. In children with spinal muscular atrophy, early provision of noninvasive ventilation may provide a tool for airway clearance, and the flexibility to be used as a method of supporting ventilation in more severe respiratory episodes, without increasing the strain of caring for a child with a severe physical disability [8]. Knowing that patients with spinal muscular atrophy have predominant expiratory muscle weakness, and patients with Duchenne muscular dystrophy experience inspiratory and expiratory muscle failure in parallel, provides us with the knowledge that prescription of treatment should be tailored to individual diagnosis. I have also evaluated noninvasive methods of secretion clearance; intrapulmonary percussive ventilation and mechanical insufflation/exsufflation have a role in the mobilisation and expectoration of secreations. Airway clearance techniques should be titrated to the individual depending on the amount of respiratory muscle weakness. Due to financial factors, treatments should start simply and then be modified as each technique becomes less effective, thus improving patient care. If secretions are not mobilised by increasing the individual's tidal volume, intrapulmonary percussive ventilation can be considered. Mechanical insufflation/exsufflation and intrapulmonary percussive ventilation provide extra methods to assist this patient group when they become vulnerable to sputum retention. This provides neuromuscular disease patients with expert advice in airway clearance management.

\section{REFERENCES}

1 Toussaint M, Chatwin M, Soudon P. Mechanical ventilation in Duchenne patients with chronic respiratory insufficiency: 
clinical implications of 20 years published experience. Chron Respir Dis 2007; 4: 167-177.

2 Chatwin M, Simonds AK. Expiratory versus inspiratory muscle strength in spinal muscular atrophy and other neuromuscular disorders. Am J Respir Crit Care Med 2004; 169: Suppl. 2, A443.

3 Chatwin M, Ross E, Hart N, Nickol AH, Polkey MI, Simonds AK. Cough augmentation with mechanical inexsufflation in patients with neuromuscular weakness. Eur Respir J 2003; 21: 502-508.

4 Chatwin M, Simonds AK. Randomised crossover trial of physiotherapy plus mechanical in-exsufflation versus physiotherapy in NIV dependent neuromuscular patients with an acute respiratory tract infection. Eur Respir J 2006; 28: Suppl. 50, 762s.
5 Ward S, Chatwin M, Heather S, Simonds AK. Randomised controlled trial of non-invasive ventilation (NIV) for nocturnal hypoventilation in neuromuscular and chest wall disease patients with daytime normocapnia. Thorax 2005; 60: 1019-1024.

6 Dayer MJ, Jonville S, Chatwin M, et al. Exercise-induced depression of the diaphragm motor evoked potential is not affected by non-invasive ventilation. Respir Physiol Neurobiol 2007; 155: 243-254.

7 Nava S, Sturani C, Hartl S, et al. End-of-life decision-making in respiratory intermediate care units: a European survey. Eur Respir J 2007; 30: 156-164.

8 Chatwin M, Simonds AK. Pilot study of preventative use of non-invasive ventilation (NIV) in type I and II spinal muscular atrophy (SMA). Am J Respir Crit Care Med 2006; 173: Suppl. 3, A753. 